\title{
The First Case of Diarrhoea in Tibetan Sheep, Ovis aries, Caused by Balantidium coli in the Qinghai Tibetan Plateau Area, China
}

\author{
Ying-Na Jian', Ge-Ping Wang', Xiu-Ping Li, Xue-Yong Zhang*, Li-Qing Ma* \\ State Key Laboratory of Plateau Ecology and Agriculture, Qinghai University, Qinghai Academy of Animal Sciences and Veterinary Medicine, Qinghai \\ University, Xining City, 810016, Qinghai Province, P. R. China
}

\begin{abstract}
This study was carried out to determine the pathogen-causing diarrhoea in sheep Ovis aries in the Qinghai Tibetan Plateau Area, China. A trophozoite was identified as species of ciliate alveolates infecting the sheep based on morphological characteristics examined by microscope. It was mostly spherical, colourless and transparent, with many vesicles. Macronucleus and contractile vacuoles could not be distinguished. Size of the trophozoite was 80-180 $\times 70-150 \mu \mathrm{m}$ and its surface was covered with cilia. Molecular analysis based on sequences of 18S rRNA and ITS genes confirmed the ciliate species as Balantidium coli. According to the literature, there have been many epidemiological investigations of $B$. coli infection in pigs, monkeys and humans. To our knowledge, this was the first report of $B$. coli infections in sheep in the Qinghai Tibetan Plateau Area of China, or eleswhere around the world. Importantly, the sheep case was rare but raised our concern that $B$. coli may spread across species and expand its host range.
\end{abstract}

Key words: Balantidium coli, sheep, morphological characteristics, molecular method, Qinghai Tibetan Plateau, China

\section{INTRODUCTION}

Balantidium coli (B. coli, former name, new/novel generic name Neobalantidium coli, N. coli) is an important zoonotic protozoan parasite, which can infect a wide variety of hosts, including pigs (domestic and wild), humans and non-human primates, rodents, cattle, camels and horses, causing the disease balantidiasis [1]. Balantidiasis is also known as B. coli infection and is treated as a neglected tropical zoonotic disease. B. coli is parasitic and infects the small intestine, rectum, and caecum of the host. The manifestations of serious infection include severe diarrhoea and other digestive diseases [2]. To our knowledge, pigs are an important reservoir host and humans and other animals can be infected through the faecal-oral route by food and/or water contaminated with B. coli [3]. There are some epidemiological reports about the prevalence of $B$. coli infections in pigs [4-6] in China; however, there are no positive data about $B$. coli infections in sheep (goats) except one case in

- Received 23 May 2018, revised 15 October 2018, accepted 19 October 2018.

*Corresponding authors (maliq67@hotmail.com; zhang_xyong@163.com)

${ }^{\dagger}$ These authors contributed equally to this work.

(c) 2018, Korean Society for Parasitology and Tropical Medicine

This is an Open Access article distributed under the terms of the Creative Commons Attribution Non-Commercial License (http://creativecommons.org/licenses/by-nc/4.0) which permits unrestricted non-commercial use, distribution, and reproduction in any medium, provided the original work is properly cited. which $B$. coli trophozoites were found within the lymphatic ducts of the gastric lymph node and the abdominal submucosa of the zoo barbary sheep. Unexpectedly, there were no B. coli trophozoites or cysts detected in the faeces [7]. According to the epidemiological report of gastrointestinal protozoan and helminthic infections in cattle, buffaloes, goats and sheep in southern Rajasthan of India, B. coli was found only in cattle $(10.76 \%)$, and not detected in goats and sheep [8].

Therefore, the present study was performed by morphological and molecular methods to identify the species of ciliate alveolates that was rarely found in Tibetan sheep with diarrhoea in the Qinghai Tibetan Plateau Area (QTPA), China. It also raised our concern about this parasite spreading across species and expanding its host range and becoming a zoonotic threat to public health.

\section{CASE RECORD}

One faecal sample from a 2.5-year-old male Tibetan sheep (Ovis aries) with diarrhoea was collected and tested in the laboratory. The faecal sample was further processed and purified by the sucrose density gradient centrifugation method. Then, the purified sample was checked microscopically with an optical microscope and captured with a DP 25 digital camera 
(NIKON, Tokyo, Japan).

The slides were observed with an optical microscope, and it was found that the trophozoites could be identified as the species of ciliate alveolates based on morphological characteristics, which were mostly spherical, colourless and transparent, with many round vesicles. The macronuclear and contractile vacuoles cannot be distinguished. The size of the trophozoite was $80-180 \times 70-150 \mu \mathrm{m}$. Surface was covered with cilia. The trophozoites spread quickly and the sheep had serious diarrhoea and high-density infections (Fig. 1A). It was also observed the trophozoite was ovoid or spherical in shape, but it became more rounded (Fig. 1B, C). We observed the trophozoites moving very quickly (Fig. 1D, E). They featured an oral opening at the anterior end and were covered with cilia on the whole external surface, and one macro nucleus were present (Fig. 1F, G).

The total genomic DNA was extracted from the stool sample using the QIAamp DNA Stool Mini Kit (Qiagen, Hilden, Germany) according to the manufacturer's instructions. PCR was performed to amplify the 18S rRNA gene and ITS gene with following primers: B. coli 18SF: 5'-TTGTCGCGATAAATCATC-
CA-3' and B. coli 18SR: 5'-GCAGGTTAAGGTCTCGTTCG-3' (product $=980 \mathrm{bp}, \mathrm{Tm}=55^{\circ} \mathrm{C}$ ), B. coli ITSF: $5^{\prime}$-CCTGCGGAAGGATCATTAAC-3' and B. coli ITSR: 5'-GCTTTAATTCAGCGGGTCAT- $3^{\prime}$ (product $=420 \mathrm{bp}, \mathrm{Tm}=55^{\circ} \mathrm{C}$ ). The amplification products were analyzed by electrophoresis by loading $10 \mu \mathrm{l}$ of PCR products on a 1.5\% agarose gel (Biowest Regular Agarose G-10, manufacturerd to specifications distributed by Gene Company Ltd., Hong Kong, China, origin: Spain) stained with ExRed nucleic acid electrophoresis dye (Beijing Zoman Biotechnology, Beijing, China), Gels were observed and images captured using a WD-9413B gel imaging analysis system (Beijing Liuyi Biotechnology, Beijing, China).

Direct sequencing was performed on the positive PCR products by the Beijing GENEWIZ Company (Beijing, China). The obtained sequences were then aligned with reference sequences available in the GenBank database using BLAST (http:// blast.ncbi.nlm.nih.gov/Blast.cgi). Phylogenetic relationships of Balantidium spp. were constructed by the MEGA 5.05 software with the neighbour-joining method, which was performed using the Kimura 2-parameter model and 2,000 bootstrap replicates.
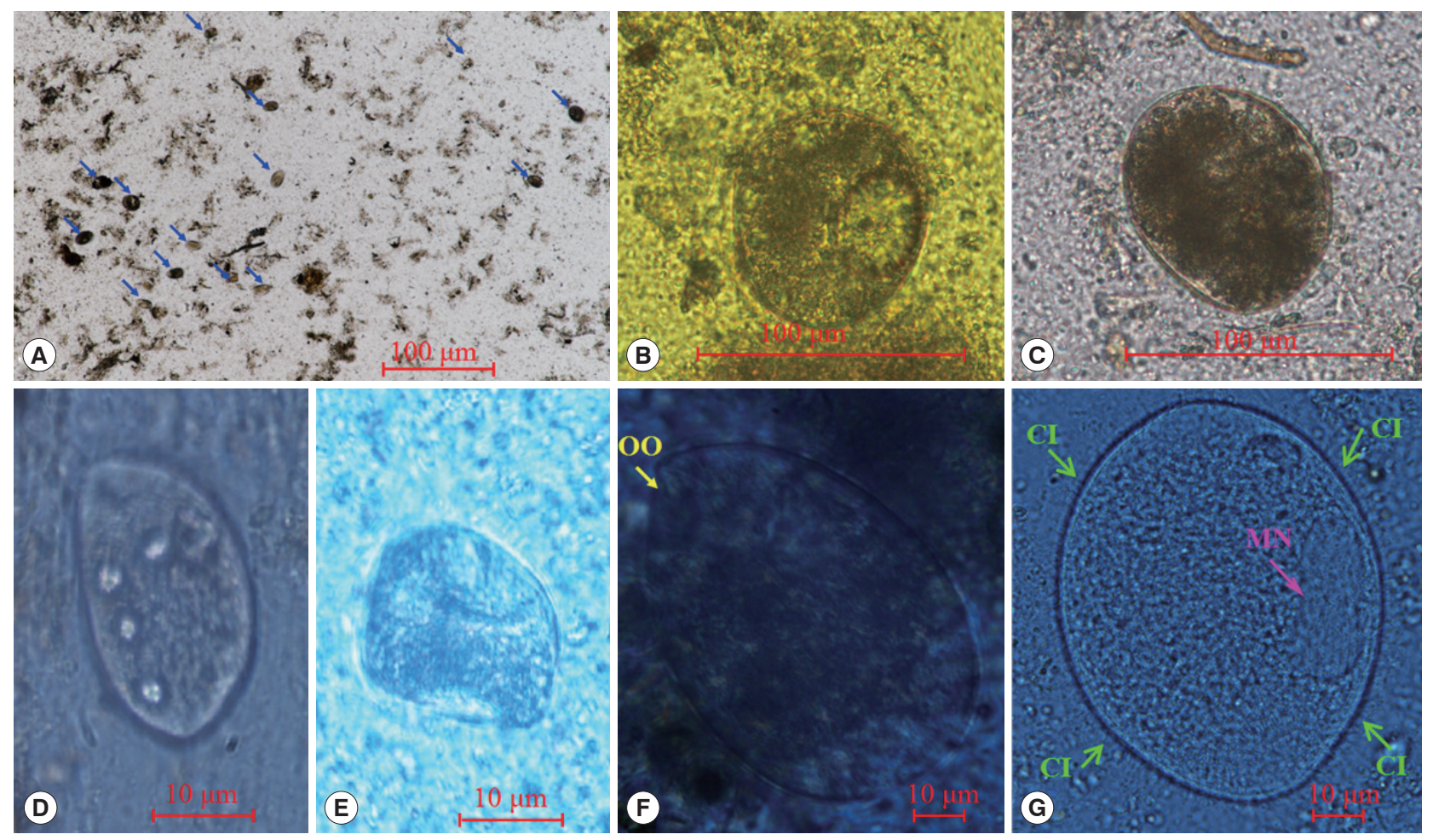

Fig. 1. The trophozoite and cyst forms of Balantidium coli observed in sheep faecal samples. (A) Trophozoites of $B$. coli $(\times 4)$ (blue arrows); $(\mathrm{B}, \mathrm{C})$ Cysts of $B$. coli $(\times 20)$; (D, E) The movement state of B. coli $(\times 40) ;(\mathrm{F}, \mathrm{G})$ The oral opening (OO, yellow arrow), cilia $(\mathrm{Cl}$, green arrows) and macro nucleus (MN, pink arrow) of the trophozoites of $B$. coli $(\times 100)$ detected in direct examination of sheep faecal samples. 
The species was identified as B. coli following the amplification of the fragments of the 18S rRNA gene (GenBank accession No. MH206219) and ITS gene (MH223458), which showed $100 \%$ homology to the $18 \mathrm{~S}$ rRNA gene of $N$. coli (KJ170367/JQ0 73305/11/12/15/16/22/31/33/35) and B. coli (GQ903678/ AF029763/EU680309), and 99\% homology to the ITS gene of N. coli (JQ073346/47/48/50/51/53/54/55/56/59/72/73/74/76/8 0) and B. coli (EU680315/6, HF545448, JF444758, AM982724), when blasted against the NCBI database. Phylogenetic reconstructions with the published Balantidium 18S rRNA gene and the ITS gene sequences confirmed that this isolate was closely related to B. coli (Fig. 2). Although the phylogenetic trees based on the $18 \mathrm{~S}$ rRNA gene and ITS gene did not show obvious geographical distribution specificity and host specificity of the Balantidium isolates, the isolate from sheep in this study was branched separately from other Balantidium isolates located far distances from China (Fig. 2). Conversely, the isolate from sheep was placed into a sub-clade with primates and domestic pigs from other countries (Fig. 2).

\section{DISCUSSION}

Balantidiasis is an infectious disease commonly occurring in pigs worldwide, but also a serious zoonosis. Pigs are known to be $B$. coli reservoir hosts, which not only causes economic losses in the pig industry, but also exposes humans directly or indirectly resulting in co-infections [1]. B. coli has a wide range of hosts, such as humans, primates, wild and domestic pigs, camels and guinea pigs [9]. B. coli infections were most prevalent in pigs, with 29.6\% prevalence in Swiss pigs [10]; 36.9\% prevalence in pigs in Hunan province of subtropical China [3]; a $15.8 \%$ prevalence in Cambodian pigs [11]; a $64.7 \%$ prevalence in pigs in rural areas of Chungcheongnam-do, Korea [12]; a $10.76 \%$ prevalence in cattle in southern Rajasthan, India [8];
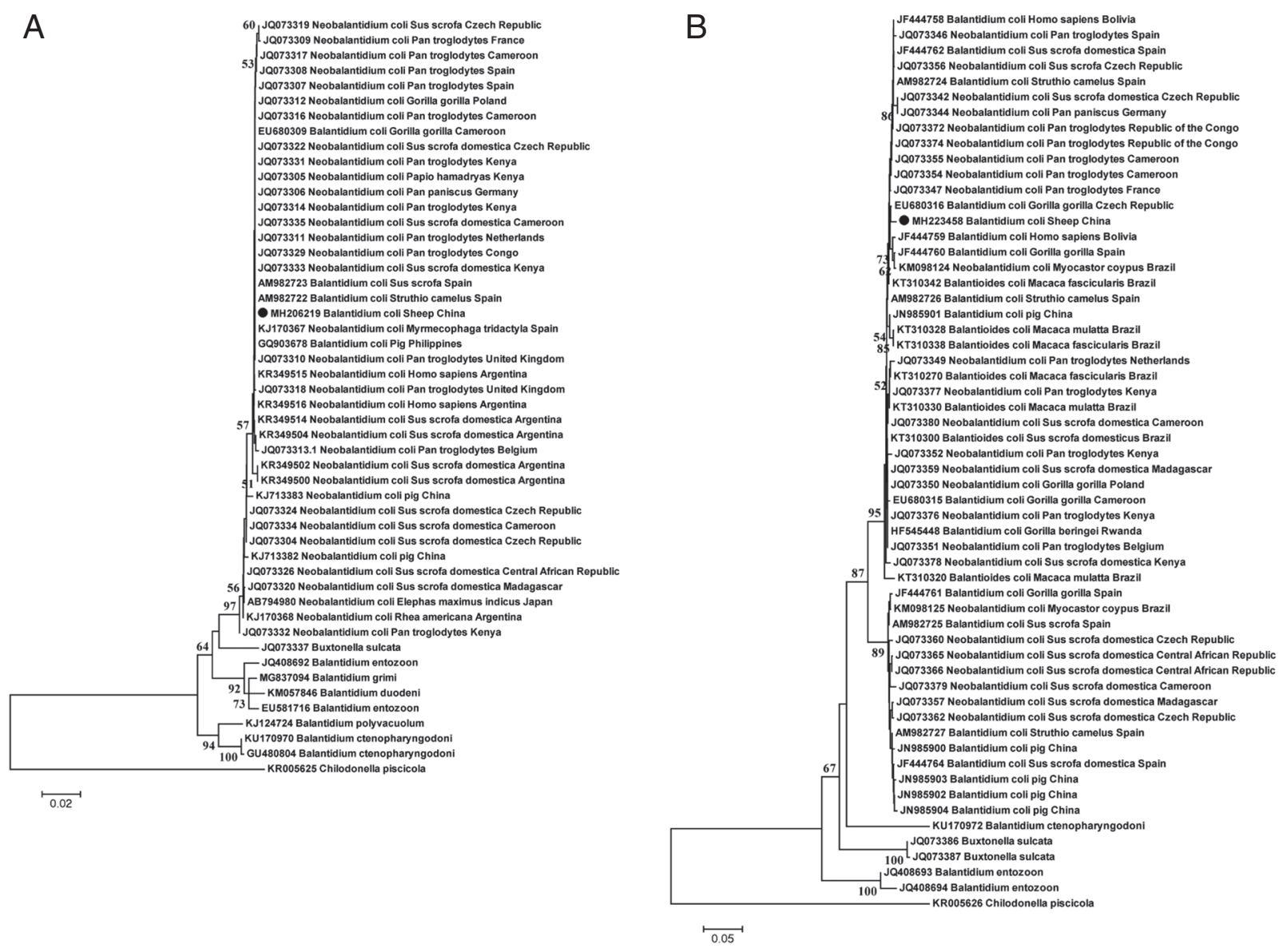

Fig. 2. Phylogenetic analysis of Balantidium based on the sequence of partial 18S rRNA gene (A) or ITS gene (B). Chilodonella piscicola was used as an outgroup. The black circle represents the $B$. coli in this study. The phylogenetic tree was constructed by using the neighbour-joining method, and the Kimura 2-parameter model with 2,000 bootstrap replicates. 
a 2.52\% prevalence in cattle from Ludhiana district of Punjab, India [13]; an 18.3\% prevalence in donkeys from Lahore, Pakistan [14]; a 52.6\% prevalence in captive African great apes [15]; a $62 \%$ prevalence in Mole National Park, Ghana [16]; a 64.2\% prevalence in bred rhesus monkeys in Guangxi Zhuang Nationality Autonomous Region, southern China [5]; and a 0.7\% prevalence in humans in Panama [17]. However, data were lacking on B. coli infections in sheep and goats, so the present study is rare but thought-provoking.

In this study, trophozoites were found in the dilute faecal sample from the Tibetan sheep, which had severe diarrhoea and weight loss. For these symptoms in sheep, the bacterial and viral pathogens are usually considered to be the first and chief "culprits". Parasites are the last to be considered, which results in missing or delaying the best treatment time. In the most cases, the diarrhoea-caused parasites are always parasitic in the intestines of the hosts, and the trophozoites of the parasites are produced in the intestines and excreted in the faeces. Therefore, it is difficult to accurately identify parasitic trophozoites. In this study, we were able to identify the trophozoites as ciliate alveolates morphologically. The size of trophozoite $(80-180 \times 70-150 \mu \mathrm{m})$ was larger than it was in Schuster and Ramirez-Avila's study $(30 \times 25 \mu \mathrm{m}$ to $150 \times 120 \mu \mathrm{m})$, but it was ovoid or spherical in shape, featured an oral opening at the anterior end and a cytopyge at the posterior end. The trophozoite was covered with cilia on the whole external surface and had one micro and one macro nucleus $[1,18,19]$. Meanwhile, the cyst form was spherical in shape and the diameter of the cyst (50 to $70 \mu \mathrm{m}$ ) was also larger (40 to $60 \mu \mathrm{m})[1,18,19]$. There were minor differences in the morphological description and comparison analysis, which is rapid and more convenient advantages; however, it is not a very accurate method to identify the exact parasite species. To further determine the exact parasite species, the molecular identification was essential.

The $18 \mathrm{~S}$ rRNA gene and ITS gene loci sequences were obtained, and the extensive evaluation and polyphyletic analysis of $B$. coli were performed. In the study of Pomajbikova et al. [20], the 2 gene markers SSrDNA and ITS1-5.8SDNA-ITS2 trees all showed the same phylogenetic diversification at the genus level of $B$. coli, which originated from 11 species of captive and wild primates, domestic pigs and wild boars [20]. In the present study, we also analysed our sheep isolate together with the reference sequences of $B$. coli and $N$. coli from humans, nonhuman primates and domestic and wild pigs using the phylogenetic markers: $18 \mathrm{~S}$ rRNA gene and ITS gene. Our phylogenetic analyses showed the same phylogenetic character of B. coli and N. coli gathered into a large clade at the genus level of the tree constructed based on the ITS gene sequences, which appeared to be phylogenetically distinct from B. entozoon. In the B. coli and N. coli clade, the sheep isolate was not clustered with closely related Chinese isolates, but clustered with isolates from other countries, which implied that it may be geographical isolate formed from an adaptive mutation based on the evolutionary analysis of the 18S rRNA gene and ITS gene. Moreover, the sheep isolate was loosely related with humans and non-human primates, which suggested that there was a zoonotic risk of infections. Clearly, balantidiasis is a tropical disease, which is prevalent in tropical and subtropical regions based on epidemiological data analysis [21]. Therefore, the occurrence of balantidiasis is less frequent in cold plateau areas, which was the reason that that $B$. coli was not considered at first. Recently, the development of transportation in the cattle and sheep may increase transmission of the disease. On the other hand, there were many migratory birds in the QTPA (especially Qinghai Lake), and the birds were infected and carried the B. coli pathogens $[22,23]$. Therefore, humans and other animals may be infected by migrating birds excreting faeces.

In conclusion, this is the first report of $B$. coli infection in sheep in the QTPA, China, which indicates that presence of $B$. coli, another new parasitic pathogens causing sheep diarrhoea and it may spread in this area. Therefore, further studies are needed to determine the prevalence of $B$. coli infections in sheep, goats, yaks and birds in the same environment and areas. Although this sheep case was rare, it raised our public health concerns that $B$. coli may spread across species and expand its host range.

\section{ACKNOWLEDGMENTS}

The authors would like to express their gratitude and appreciation to the staff working at the sheep farm. This study was supported by the National Wool-purpose Sheep Industry Technology System Project (CARS-39-06B) and the Research and Demonstration of Diagnosis and Prevention and Control Technology of Major Diseases of Livestock (2017-NK-110).

\section{CONFLICT OF INTEREST}

The authors declare that they have no conflict of interest related to this work. 


\section{REFERENCES}

1. Schuster FL, Ramirez-Avila L. Current world status of Balantidium coli. Clin Microbiol Rev 2008; 21: 626-638.

2. McLeod C, Smith P, McGuinness SL, Francis JR, Baird RW. Human case of Balantidium infection in Australia. Pathology 2015; 47: 603-604.

3. Yin DM, Lv CC, Tan L, Zhang TN, Yang CZ, Liu Y, Liu W. Prevalence of Balantidium coli infection in sows in Hunan province, subtropical China. Trop Anim Health Prod 2015; 47: 1637-1640.

4. Lai M, Zhou RQ, Huang HC, Hu SJ. Prevalence and risk factors associated with intestinal parasites in pigs in Chongqing, China. Res Vet Sci 2011; 91: e121-e124.

5. Li HL, Li Q, Dong L, Li J, Zou FC, Zhang L. Prevalence of Balantidium coli infection in Bred Rhesus Monkeys (Macaca mulatta) in Guangxi, southern China. Iran J Parasitol 2014; 9: 125-128.

6. Weng YB, Hu YJ, Li Y, Li BS, Lin RQ, Xie DH, Gasser RB, Zhu $\mathrm{XQ}$. Survey of intestinal parasites in pigs from intensive farms in Guangdong Province, People's Republic of China. Vet Parasitol 2005; 127: 333-336.

7. Cho HS, Shin SS, Park NY. Balantidiasis in the gastric lymph nodes of Barbary sheep (Ammotragus lervia): an incidental finding. J Vet Sci 2006; 7: 207-209.

8. Choubisa SL, Jaroli VJ. Gastrointestinal parasitic infection in diverse species of domestic ruminants inhabiting tribal rural areas of southern Rajasthan, India. J Parasit Dis 2013; 37: 271-275.

9. Nakauchi K. The prevalence of Balantidium coli infection in fiftysix mammalian species. J Vet Med Sci 1999; 61: 63-65.

10. Schubnell F, von Ah S, Graage R, Sydler T, Sidler X, Hadorn D, Basso W. Occurrence, clinical involvement and zoonotic potential of endoparasites infecting Swiss pigs. Parasitol Int 2016; 65: 618-624.

11. Schar F, Inpankaew T, Traub RJ, Khieu V, Dalsgaard A, Chimnoi W, Chhoun C, Sok D, Marti H, Muth S, Odermatt P. The prevalence and diversity of intestinal parasitic infections in humans and domestic animals in a rural Cambodian village. Parasitol Int 2014; 63: 597-603.

12. Ismail HA, Jeon HK, Yu YM, Do C, Lee YH. Intestinal parasite infections in pigs and beef cattle in rural areas of Chungcheongnam-do, Korea. Korean J Parasitol 2010; 48: 347-349.

13. Singh NK, Singh H, Jyoti, Haque M, Rath SS. Prevalence of parasitic infections in cattle of Ludhiana district, Punjab. J Parasit Dis 2012; 36: 256-259.

14. Khan A, Khan MS, Avais M, Ijaz M, Ali MM, Abbas T. Prevalence, hematology, and treatment of balantidiasis among donkeys in and around Lahore, Pakistan. Vet Parasitol 2013; 196: 203-205.

15. Pomajbíková K, Petrželková KJ, Profousová I, Petrášová J, Modrý D. Discrepancies in the occurrence of Balantidium coli between wild and captive African great apes. J Parasitol 2010; 96: 11391144.

16. Ryan SJ, Brashares JS, Walsh C, Milbers K, Kilroy C, Chapman CA. A survey of gastrointestinal parasites of olive baboons (Papio anubis) in human settlement areas of Mole National Park, Ghana. J Parasitol 2012; 98: 885-888.

17. Sandoval NR, Rios N, Mena A, Fernandez R, Perea M, ManzanoRomán R, Santa-Quiteria JA, Hernández-Gonzalez A, Siles-Lucas M. A survey of intestinal parasites including associated risk factors in humans in Panama. Acta Trop 2015; 147: 54-63.

18. Kapur P, Das AK, Kapur PR, Dudeja M. Balantidium coli liver abscess: first case report from India. J Parasit Dis 2016; 40: 138-140.

19. Hazarika M, Pai HV, Khanna V, Reddy H, Tilak K, Chawla K. Rare case of polymicrobial keratitis with Balantidium coli. Cornea 2016; 35: 1665-1667.

20. Pomajbíková K, Oborník M, Horák A, Petrželková KJ, Grim JN, Levecke B, Todd A, Mulama M, Kiyang J, Modrý D. Novel insights into the genetic diversity of Balantidium and Balantidiumlike cyst-forming ciliates. PLoS Negl Trop Dis 2013; 7: e2140.

21. Kline K, McCarthy JS, Pearson M, Loukas A, Hotez PJ. Neglected tropical diseases of Oceania: review of their prevalence, distribution, and opportunities for control. PLoS Negl Trop Dis 2013; 7: e1755.

22. Marietto-Goncalves GA, Fernandes TM, Silva RJ, Lopes RS, Andreatti Filho RL. Intestinal protozoan parasites with zoonotic potential in birds. Parasitol Res 2008; 103: 1237-1240.

23. Otegbade AC, Morenikeji OA. Gastrointestinal parasites of birds in zoological gardens in south-west Nigeria. Trop Biomed 2014; 31: $54-62$. 
